\title{
COMMENTARY
}

\section{The Case against Universal Varicella Vaccination}

\author{
Gary S. Goldman \\ Medical Veritas International Inc., Pearblossom, California, USA
}

In 1995, the United States became the first country to implement a Universal Varicella Vaccination Program. Several questions remain: Is the varicella (chickenpox) vaccine needed? Is it cost effective as a routine immunization for all susceptible children? Or is it more beneficial for the disease to remain endemic so that adults may receive periodic exogenous exposures (boosts) that help suppress the reactivation of herpes zoster (shingles). In addition, as vaccination coverage becomes widespread, does loss of immunologic boosting cause a decline in vaccine efficacy and result in a reduced period of immunity? Scientific literature regarding safety of the varicella vaccine and its associated cost-benefit analysis have often reported optimistic evaluations based on ideal assumptions. Deleterious outcomes and their associated costs must be included when making a circumspect assessment of the Universal Varicella Vaccination Program.

Keywords Herpes Zoster, Universal Varicella Vaccination Program, Varicella Immunization, Varicella Vaccine Adverse Reactions, Varicella Vaccine Efficacy

\section{BACKGROUND}

Varicella (chickenpox) and herpes zoster (shingles) both derive from the varicella-zoster virus (VZV). Varicella is typically a benign disease characterized by a rash that appears in crops, progressing from macules to papules, vesicles, pustules, and eventually to crusted lesions - all of which may be present during the peak of the clinical phase. Lesions are often concentrated on the trunk, scalp and face and symptoms resolve in 7 to 10 days. Varicella is most contagious 1 to 2 days prior to rash onset. Following primary infection with varicella, the VZV goes dormant in the body's dorsal-root ganglia. When VZV immunity declines below a certain threshold level, the virus can reactivate in the secondary infection, herpes zoster (HZ). Individuals have a $20 \%$ chance of developing HZ during their lifetime. Unlike chickenpox, $\mathrm{HZ}$ is often confined to one or several adjacent der-

Received 10 May 2006; accepted 6 June 2006.

Address correspondence to Gary S. Goldman, $\mathrm{PhD}$, Medical Veritas International Inc., P.O. Box 847, Pearblossom, CA 93553, USA. E-mail: pearblossominc@aol.com matomes. Postherpetic neuralgia, or persistent pain following the rash, occurs in $20 \%$ of patients.

Based on 5-year data prior to vaccine licensure, there was a mean of 4 million cases, 11,000 hospitalizations, and 100 deaths ( 50 children/50 adults) attributed to varicella annually. Approximately 1 million cases of $\mathrm{HZ}$ occurred annually, representing about $75 \%$ of medical costs due to VZV. Although HZ can occur at any age in individuals with a previous history of varicella or who have received the varicella vaccine, the majority of cases occur among adults aged 50 years and older. Severity of $\mathrm{HZ}$ increases with age and in some patients produces excruciating pain.

The varicella vaccine was licensed by the U.S. Food and Drug Administration (FDA) on March 17, 1995, and thereafter the Universal Varicella Vaccination Program was implemented, whereby the Centers for Disease Control and Prevention (CDC) recommended that all healthy, susceptible children aged 12 months to 12 years receive a single dose. To monitor baseline trends in disease, the CDC funded a Varicella Active Surveillance Project (VASP) in each of three different areas: Antelope Valley (California), West Philadelphia (Pennsylvania), and Travis County (Texas). By 1999, each VASP reported incidence of varicella had dramatically declined in their respective study communities. Currently, nearly all states have enacted mandates requiring varicella vaccination for entry-level school children.

\section{INTRODUCTION}

"The Case for Universal Varicella Immunization" by Jane F. Seward and Walter A. Orenstein (2006) certainly highlights some of the perceived benefits of the universal varicella vaccination of children including decreasing morbidity and mortality from varicella (Seward et al. 2002; Nguyen, Jumaan, and Seward 2005). In response to a previous publication by Seward et al. (2002), also discussing only the benefits of varicella vaccination, Marc Brisson, W. John Edmunds et al. (2002b) stated, in part, "... the dramatic decline in incidence observed by Seward et al. might lead to a significantly increased incidence of herpes zoster (HZ) over the next 50 years ... Thus, Seward et al. report only half the story: trends in the annual age-specific incidence of $\mathrm{HZ}$ should be presented alongside the varicella data to show 
the full impact of the vaccination program on VZV disease." Although this commentary presents the other half of the story; technically, it presents the more significant issues because VZV contributes to both varicella and $\mathrm{HZ}$ (shingles) disease, with $\mathrm{HZ}$ contributing approximately three times the morbidity and five times the mortality of varicella-with a documented HZto-varicella direct cost ratio of over 4 to 1 (Nowgesic et al. 1999).

Seward et al.'s (2006) "Case for universal varicella immunization," indicates "Germany is the only country in Europe with routine childhood immunization. Varicella vaccine was incorporated into the routine immunization schedule in July 2004, as a single dose at the age of 11 to 14 months." Other countries in Europe, however, are carefully weighing both the pros and cons to decide if the cost-benefit of varicella vaccination, including potential booster vaccinations, is truly favorable.

\section{DURATION OF IMMUNITY}

Trials of the varicella vaccine in Japan demonstrated an increase in antibody titers after 20 years (Asano 1996). In Japan, however, only about 1 in $5(20 \%)$ children are vaccinated. In the varicella vaccine application for Varivax, Merck explained that a boost in antibody levels has been observed in vaccinees following exposure to natural varicella, which could account for the apparent long-term persistence of antibody levels after vaccination and noted "the duration of protection from varicella obtained using Varivax ${ }^{\circledR}$ in the absence of wild-type boosting is unknown."

Many of the trials and postlicensing studies and testing concerning vaccinees in the United States were conducted in communities where natural (wild-type) varicella incidence was still high. Thus, estimates of the vaccine effectiveness (Seward et al. 2004) as well as the duration of immunity were confounded (and overestimated) because of the immunologic boosting alluded to by Merck. As long as incidence of natural varicella remained high in the community, the concomitant exogenous exposures effectively boosted cell-mediated immunity in vaccinees that received a single dose and there were no adverse effects on the closely related herpes-zoster epidemiology.

\section{DECLINE IN VACCINE EFFICACY (VE)}

When communities experience dramatic declines in varicella incidence due to moderate to widespread vaccination coverage, interesting, but predictable, results occur that are related to loss of immunologic boosting - including decline in VE and the need for a booster dose.

When stratified by year, rather than averaged over years that include high incidence of natural varicella, a significant decline is observed in VE (especially by 2002). As anticipated, VE reached a maximum during 1999 when there were boosting effects provided by recent cases of natural varicella (sometimes referred to as a "honeymoon effect"); however, following a dramatic decline in exposures (boosts) to natural cases of varicella, VE declined rapidly and significantly in subsequent years to less than $60 \%$ by 2002 based on the analysis of varicella transmission within households (Goldman 2005a). Galil et al. (2002) reported a VE of $44 \%$ (95\% C.I. $7 \%-66 \%)$ in an outbreak among a high proportion of vaccinees at a day care. Moreover, children with previous histories of natural varicella experienced unusually high incidence rates of $\mathrm{HZ}$, approaching those found among older adults (Goldman 2003).

The fact that children having a previous history of natural varicella experience higher incidence of $\mathrm{HZ}$ relative to vaccinees seems to be a powerful argument for vaccination. However, a vaccinated child who is also exposed to either an adult with shingles or a child with natural disease, can transmit the natural VZV to other vaccinated children (Arvin and Gershon 2006). The natural or wild-type varicella dominates the attenuated Oka or vaccine strain VZV such that these vaccinees subsequently become candidates for higher incidence of $\mathrm{HZ}$ associated with those harboring natural VZV.

A booster vaccine for children aged 12 months to 12 years has already been discussed in the United States and in Germany (Arvin and Gershon 2006; Knuf et al. 2006) and as of April 5, 2006, an optional second dose has been approved by the FDA (Krause 2005).

\section{A "SHINGLES" VACCINE}

Interestingly, a "new tool" as Seward et al. label it "a newly formulated high potency VZV vaccine" has shown that it reduces $\mathrm{HZ}$ incidence by about 50\% (Oxman et al. 2005). However, offsetting this benefit is this statistic: during a period of increasing varicella vaccination, $\mathrm{HZ}$ incidence among adults increased $90 \%$, from $2.77 / 1000$ to $5.25 / 1000$ in the period 1998 to 2003 (Yih et al. 2005).

Similar high percentage increases in zoster incidence using different methodology and in a different study population were also reported by the Varicella Active Surveillance Project (VASP), conducting active surveillance of HZ in the Antelope Valley region of California since 2000 among a population of over 300,000. Zoster cases among adults aged 20 years and older reported principally by healthcare providers increased $18 \%$ from 237 cases in 2000 to 279 in 2001 with increases in nearly every 10 -year age group from 20-29 through 60-69. Generally, young adults, who previously received the most exogenous exposures (boosts) in the prelicensure era, experienced the greatest percentage increase in case reports relative to the older adults. A total of $370 \mathrm{HZ}$ case reports among adults in 2002 represented an increase of $32.6 \%$ and $56.1 \%$ over those cases reported in 2001 and 2000, respectively (Goldman 2005b). The incidence rate ratio of 1.4 (95\% C.I. 1.2-1.7) demonstrates a statistically significant increase during 2000 to 2002.

Historically, the medical community thought that an agerelated decline in immunity was the principal reason for the observed increase in HZ with advancing age. As early as 1965, Hope-Simpson, following a 16-year study of HZ in Cirencester, England, first suggested, "The peculiar age distribution of zoster 
may in part reflect the frequency with which the different age groups encounter cases of varicella and because of the ensuing boost to their antibody protection have their attacks of zoster postponed" (Hope-Simpson 1995). Based on accumulating evidence that (a) $\mathrm{HZ}$ incidence is substantially higher in adults without children than those with children (Brisson et al. 2002a; Thomas, Wheeler and Hall 2002); (b) physicians exhibit 1/2 to $1 / 8$ the HZ incidence of the general population (Terada et al. 1965); and (c) increased immunity on the cellular level was found among individuals having close exposure to varicella (Arvin Koropchak, and Wittck 1983; Arvin 1992, 1996; Gershon et al. 1996), it became clear that periodic exogenous reexposures to varicella infections played a significant role in boosting cellmediated immunity to levels that help suppress $\mathrm{HZ}$ reactivation. The increase in $\mathrm{HZ}$ with advancing age was then understood to be attributable to adults having decreased contact with children, especially as many transitioned in their role from parents toward grandparents.

In the absence of exogenous boosts, the postlicensure data from communities with moderate to widespread vaccination coverage indicate that asymptomatic endogenous reactivations self-limit HZ incidence to approximately 5.5/1000 among adults and children with histories of natural varicella. This upper limit is not unexpected; the rationale being that it is approximately the same as the prelicensure rate found among adults 50 to 59 who generally had no young children in their household and thus had few opportunities for exogenous exposures. The steep increase in $\mathrm{HZ}$ incidence among those in their 60s, 70s, and older, demonstrates that an age-related decline in immunity predominates only in the elderly.

When increases in $\mathrm{HZ}$ following universal varicella vaccination are taken into account, the cost is $\$ 20$ to $\$ 40$ billion (at $\$ 100$ per dose) for the adult U.S. population to be vaccinated to achieve protection similar to that which occurred naturally in the community when varicella incidence was high (Brisson et al. 2000; Goldman 2005b).

\section{ADVERSE REACTIONS}

Historically, mass vaccination of adults has seldom been successful. Further, not only is there the issue of weak protective outcomes, but adults also tend to experience a higher rate of adverse vaccination effects relative to children, including a higher rate of serious adverse effects. One possible example of this type of serious event following immediately after a varicella booster, involved an adult female, age 47, employed as a research coordinator in the infectious disease unit of a medical school. She and other varicella-immune employees were recruited for a manufacturer-sponsored study aimed at detecting the boosting effect of the vaccine. Her prestudy laboratory values were completely normal prior to her receiving a first dose of vaccine in March, 2001. Shortly following the second dose administered in May, she developed diarrhea and by November 2001, she was quite ill with serious intestinal difficulties. She was diagnosed with a rare collagenous colitis that was autoimmune in nature. While her lymphocytes decreased from $25 \%$ to $14 \%$, her total white count, neutrophils and eosinophils increased. She remains disabled and, because the change in her health was clearly associated with the second vaccine dosing, the event was reported to the Vaccine Adverse Events Reporting System (VAERS). Though causality was not proven in this case, it is suggestive of a serious side effect of the vaccine.

Varicella vaccination is generally considered safe but there are usually no prescreening tests to determine whether an adverse reaction is likely to occur (Poser 2003). The literature contains a surprising number of adverse reactions following varicella vaccination (Ravkina and Matsevich 1970; Sunaga et al. 1995; Singer et al. 1999; Gerecitano, Friedman-Kien, and Chazen 1997; Sakaguchi et al. 1997; Naruse et al. 1993; Lee, Komp, and Andiman 1986; Wrensch et al. 2001; Naseri, Good, and Cunningham 2003; Esmaeli-Gutstein and Winkelman 1999; Schwab and Ryan 2004; Bronstein et al. 2005; Binder et al. 2005; Grossberg et al. 2006) including vaccine-strain zoster in children and adults (Matsubara et al. 1995; Hammerschlag et al. 1989). Because follow-up is not conducted, it may be argued that some reports may not be attributed to or associated with vaccination and therefore the true rate of adverse events is essentially unknown. The mean of 2350 reports/year attributed to varicella vaccine is based on 20,004 VAERS reports filed from May, 1995, through December, 2003. The first report, VAERS (ID 74221) on May 26, 1995, was issued for a 3.5-year-old boy from Georgia who had no preexisting conditions and was vaccinated on May 12, 1995. He developed convulsions the following day, was hospitalized, and reportedly recovered.

A total of 8937 (44.7\%) of the 20,004 VAERS reports filed following varicella vaccine licensure were considered serious, of which $52(0.3 \%)$ presumably resulted in death, $194(1.0 \%)$ were life-threatening, 7845 (39.2\%) involved an emergencyroom visit, and 846 (4.2\%) were hospitalized. Many physicians consider vaccination extremely safe and parents or patients are not fully informed regarding potential adverse outcomes. Because the varicella disease is relatively benign, only a few serious adverse reactions might offset the intended benefits. Serious reports associated with varicella vaccine were reported to VAERS at a rate of approximately 1000 reports/year (8937 total serious reports/8.5 years). Although the specific reporting efficiencies for various reports of adverse events associated with varicella vaccination are unknown (Singleton et al. 1999), the above figures must be multiplied by factors of 10 to 20 if it is assumed that typically only $5 \%$ to $10 \%$ of the true reactions are reported to VAERS.

The Oxman et al. (2005) study considered adverse effects in one-sixth of the subjects during 42 days following vaccination. With reference to this substudy, Kaufman (2005) states, "Extrapolating the results to 19,273 subjects in the whole treatment group, this group had 132 more cases $(0.7 \%)$ of one or more serious adverse events, and 4,677 more cases (24\%) of one or more adverse events than the placebo group." Interestingly, 
ignoring the impact of the medical costs associated with these adverse reactions, the cost to prevent one case of $\mathrm{HZ}$ is $\$ 5900$ at $\$ 100$ per dose. This figure is based on the determination that 59 individuals were treated for each $\mathrm{HZ}$ case prevented. The number needed to treat (NNT) is obtained by dividing the 19,000 vaccinated individuals by the $327 \mathrm{HZ}$ cases that were prevented (642 HZ cases occurring in the placebo group minus $315 \mathrm{HZ}$ cases occurring in the treatment group) (Oxman et al. $2005)$. Similarly, the vaccination cost is $\$ 36,000$ to prevent one case of post herpetic neuralgia (PHN) based on an NNT of 360 $[19,000 /(80-27)]$. Excluding mild cases (pain scores 3 and below) and noting that the majority of PHN cases were reported among the elderly, the NNT approaches 1000 vaccinations at a cost of $\$ 100,000$ to prevent one case of moderate to severe PHN among adults in their 60s.

The 50 deaths (out of 4 million) per year that varicella causes in children is quite low (Seward and Orenstein 2006). In perspective, a person has a greater chance of dying by being struck by lightning in the United States (mean of 90 cases annually in the United States) (Curran and Holle 1997) than of a child's dying from contracting varicella.

\section{CONCLUSIONS}

With regard to the reporting in VAERS, it is true that causality is rarely proven; however, the timing of a disease closely following a vaccination and lack of any other obvious cause are most often the only criteria of potential significance for a suspected adverse reaction. A temporal relationship was the only criteria used in the postmarketing evaluation by Black et al. (1999). Admittedly, the spontaneous reporting in general and VAERS in particular are unreliable. Thus, it is illogical for the FDA or CDC to acknowledge these limitations, yet state that VAERS serves "to reassure the general public concerning the safety of a new vaccine" (Zhou et al. 2003) — basing assessment of the safety of the varicella vaccine only on an analysis of VAERS (Wise et al. 2000). Sophisticated safety assessments using reliable methodology have never been performed and these should precede any mass vaccination program.

In communities with widespread varicella vaccine coverage, at least three initial assumptions used to justify the U.S. Universal Varicella Vaccination Program and its cost-benefit analysis (Lieu 1994), are no longer valid: (1) a single dose provides lifelong immunity; (2) there is no immunologically-mediated link between varicella incidence and $\mathrm{HZ}$ incidence; and (3) the vaccine is safe. As deleterious effects became quantified, updated cost analysis by F. Zhou (2005) of the National Immunization Program (NIP) that excluded increased HZ in persons with a history of varicella due to reduced exposure to varicella, but included $\mathrm{HZ}$ in vaccinees and outbreak management costs concluded "the two-dose program may not be cost effective." Thus, countries should take a circumspect view when evaluating the cost-benefit of universal varicella vaccination to determine if it is worth the risk of offsetting the natural balance of varicella and herpes zoster in their communities and nations and embarking upon a program that introduces continual disease and (expensive) treatment cycles.

\section{REFERENCES}

Arvin, A. M. 1992. Cell-mediated immunity to varicella-zoster virus. J. Infect. Dis. 166(Suppl. 1):S35-S41.

Arvin, A. M. 1996. Varicella-zoster virus. Clin. Microbiol. Rev. 9:361-381.

Arvin, A., and A. Gershon. 2006. Control of varicella: Why is a two-dose schedule necessary? Pediatr. Infect. Dis. J. 25:475-476.

Arvin, A. M., C. M. Koropchak, and A. E. Wittek. 1983. Immunologic evidence of reinfection with varicella-zoster virus. J. Infect. Dis. 148:200-205.

Asano, Y. 1996. Varicella vaccine: The Japanese experience. J. Infect. Dis. 174(Suppl.3):S310-S313.

Binder, N. R, G. N. Holland, S. Hosea, and M. L. Silverberg. 2005. Herpes zoster ophthalmicus in an otherwise-healthy child. J. AAPOS 9:597-598.

Black, S., H. Shinefield, P. Ray, E. Lewis, J. Hansen, J. Schwalbe, P. Coplan, R. Sharrar, H. Guess. 1999. Postmarketing evaluation of the safety and effectiveness of varicella vaccine. Pediatr. Infect. Dis. 18:10411046.

Brisson, M., W. J. Edmunds, N. J. Gay, B. Law, and G. De Serres. 2000. Modelling the impact of immunization on the epidemiology of varicella zoster virus. Epidemiol. Infect. 125:651-669.

Brisson, M., N. J. Gay, W. J. Edmunds, and J. J. Andrews. 2002a. Exposure to varicella boosts immunity to herpes-zoster: Implications for mass vaccination against chickenpox. Vaccine 20:2500-2507.

Brisson, M, W. J. Edumunds, N. J. Gay, and E. M. Miller. 2002b. Varicella vaccine and shingles. [Letter to Editor]. JAMA 287:2211.

Bronstein, D. E, J. Cotliar, J. K. Votava-Smith, M. Z. Powell, M. J. Miller, and J. D. Cherry. 2005. Recurrent papular urticaria after varicella immunization in a 15-month-old girl. Pediatr. Infect. Dis. J. 24:269-270.

Curran, E. B., and R. L. Holle. 1997. Lightning fatalities, injuries, and damage reports in the United States from 1959-1994. U.S. Department of Commerce, National Oceanic \& Atmospheric Association (NOAA) Technical Memorandum NWS SR-193. Available online at http://www.nssl.noaa.gov/papers/ techmemos/NWS-SR-193/techmemosr193.html

Esmaeli-Gutstein, B., and J. Z. Winkelman. 1999. Uveitis associated with varicella virus vaccine. Am. J. Ophthalmol. 127:733-734.

Galil, K., B. Lee, T. Strine, C. Carraher, A. L. Baughman, M. Eaton, J. Montero, and J. Seward. 2002. Outbreak of varicella at a day-care center despite vaccination. N. Engl. J. Med. 347:1909-1915.

Gerecitano, J, A. Friedman-Kien, and G. D. Chazen. 1997. Allergic reaction to varicella vaccine. Ann. Intern. Med. 126:833-834.

Gershon, A. A., P. La Russa, S. Steinberg, N. Mervish, S. H. Lo, and P. Meier. 1996. The protective effect of immunologic boosting against zoster: An analysis in leukemic children who were vaccinated against chickenpox. J. Infect. Dis. 173:450-453.

Goldman, G. S. 2003. Incidence of herpes-zoster among children and adolescents in a community with moderate varicella vaccination coverage. Vaccine 21:4243-4249.

Goldman, G. S. 2005a. Universal varicella vaccination: Efficacy trends and effect on herpes-zoster. Int. J. Toxicol. 24:205-213.

Goldman, G. S. 2005b. Cost-benefit analysis of universal varicella vaccination in the U.S. taking into account the closely related herpes-zoster epidemiology. Vaccine 23:3349-3355.

Grossberg, R., R. Harpaz, E. Rubtcova, V. Loparev, J. F. Seward, and D. S. Schmid. 2006. Secondary transmission of varicella vaccine virus in a chronic care facility for children. J. Pediatr. 148:842-844.

Hammerschlag, M. R., A. A. Gershon, S. P. Steinberg, L. Clarke, and L. D. Gelb. 1989. Herpes zoster in an adult recipient of live attenuated varicella vaccine. J. Infect. Dis. 160:535-537.

Hope-Simpson, R. E. 1965. The nature of herpes zoster: A long-term study and a new hypothesis. Proc. R. Soc. Med. 58:9-20. 
Kauffman, J. M. 2005. New vaccine for shingles: Is prevention really better than treatment? J. Am. Physicians Surgeons 10:117.

Knuf, M., P. Habermehl, F. Zepp, W. Mannhardt, M. Kuttnig, P. Muttonen, A. Prieler, H. Maurer, H. Bisanz, N. Tornieporth, D. Descamps, and P. Willems. 2006. Immunogenicity and safety of two doses of tetravalent measles-mumpsrubella-varicella vaccine in healthy children. Pediatr. Infect. Dis. J. 25:12-18.

Krause P. R. 2005. Department of Health and Human Services, Food and Drug Administration. FDA Approval Letter, April 6, 2005. Available online at http://www.fda.gov/cber/approvltr/varmer040505L.htm

Lee, S. Y., D. M. Komp, and W. Andiman. 1986. Thrombocytopenic Purpura following varicella-zoster vaccination. Am. J. Pediatr. Hematol. Oncol. 8:7880.

Lieu, T. A., S. L. Cochi, S. B. Black, M. E. Halloran, H. R. Shinefield, and S. J. Holmes, et al. 1994. Cost-effectiveness of a routine varicella vaccination program for U.S. children. JAMA 271:375-381.

Matsubara, K., H. Nigami, H. Harigaya, and K. Baba. 1995. Herpes zoster in a normal child after varicella vaccination. Acta. Paediatr. Jpn. 37:648-650.

Naruse, H., H. Miwata, T. Ozaki, Y. Asano, J. Namazue, and K. Yamanishi. 1993. Varicella infection complicated with meningitis after immunization. Acta Paediatr. Jpn. 35:345-347.

Naseri, A., W. V. Good, and E. T. Cunningham Jr. 2003. Herpes zoster virus sclerokeratitis and anterior uveitis in a child following varicella vaccination. Am. J. Ophthalmol. 135:415-417.

Nowgesic, E., D. Skowronski, A. King, and J. Hockin. 1999. Direct costs attributed to chickenpox and herpes zoster in British Columbia-1992 to 1996. Can. Commun. Dis. Rep. (CCDR) 25:100-104.

Nguyen, H. Q., A. O. Jumaan, and J. F. Seward. 2005. Decline in mortality due to varicella after implementation of varicella vaccination in the United States. N. Engl. J. Med. 352:450-458.

Oxman, M. N., M. J. Levin, G. R. Johnson, K. E. Schmader, S. E. Straus, L. D. Gelb, et al. 2005. A vaccine to prevent herpes zoster and postherpetic neuralgia in older adults. N. Engl. J. Med. 352:2271-2284.

Poser, C. M. 2003. Neurological Complications of Vaccinations. Mealey's Litigation Report, Thimerosal \& Vaccines, April, 2003; Volume 1, Issue 10.

Ravkina, L. I., and G. R. Matsevich. 1970. Morphological changes in the central nervous system in post-vaccinal encephalomyelitis developing after chickenpox vaccination in children. Zh. Nevropatol. Psikhiatr. Im. S. S. Korsakova. 70:1465-1471.

Sakaguchi, M., T. Yamanaka, K. Ikeda, Y. Sano, H. Fujita, T. Miura, and S. Inouye. 1997. IgE-mediated systemic reactions to gelatin included in the varicella vaccine. J. Allergy Clin. Immonol. 99:263-264.

Schwab, J., and M. Ryan. 2004. Varicella zoster virus meningitis in a previously immunized child. Pediatrics 114:e273-e274.
Seward, J. F., and W. A. Orenstein. 2006. Commentary: The case for universal varicella immunization. Pediatr. Infect. Dis. J. 25:45-46.

Seward, J. F., B. M. Watson, C. L. Peterson, L. Mascola, J. W. Pelosi, J. X. Zhang, T. J. Maupin, G. S. Goldman, L. J. Tabony, K. G. Brodovicz, A. O. Jumaan, and M. Wharton. 2002. Varicella disease after introduction of varicella vaccine in the United States, 1995-2000. JAMA 287:606-611.

Seward, J. F., J. X. Zhang, T. J. Maupin, L. Mascola, and A. O. Jumaan. 2004. Contagiousness of varicella in vaccinated cases. JAMA 299:704-708.

Singer, S., C. E. Johnson, R. Mohr, and C. Holowecky. 1999. Urticaria following varicella vaccine associated with gelatin allergy. Vaccine 17:327-329.

Singleton, J. A., J. C. Lloyd, G. T. Mootrey, M. E. Salive, R. T. Chen, and VAERS Working Group. 1999. An overview of the vaccine adverse even reporting system (VAERS) as a surveillance system. Vaccine 17:2908-2917.

Sunaga, Y, A. Hikima, T. Ostuka, and A. Morikawa. 1995. Acute cerebellar ataxia with abnormal MRI lesions after varicella vaccination. Pediatr. Neurol. 13:340-342.

Terada, K, Y. Hirago, S. Kawano, and N. Kataoka. 1995. Incidence of herpes zoster in pediatricians and history of reexposure to varicella-zoster virus in patients with herpes zoster. Kansenshogaku Zasshi 69:908-912.

Thomas, S. L., J. G. Wheeler, and A. J. Hall. 2002. Contacts with varicella or with children and protection against herpes zoster in adults: A casecontrol study. Lancet 360:678-682. Available online at http://image.thelancet. com/extras/01art6088web.pdf

Wrensch, M., A. Weinberg, J. Wiencke, R. Miike, G. Barger, and K. Kelsey. 2001. Prevalence of antibodies to four herpesviruses among adults with glioma [brain tumor] and controls. Am. J. Epidemiol. 154:161-165.

Wise, R. P., M. E. Salive, M. M. Braun, G. T. Mootrey, J. F. Seward, L. G. Rider, and P. R. Krause. 2000. Postlicensure safety surveillance for varicella vaccine. JAMA 284:1271-1279.

Yih, W. K., D. R. Brooks, S. M. Lett, A. O. Jumaan, Z. Zhang, K. M. Clements, and J. F. Seward. 2005. The incidence of varicella and herpes zoster in Massachusetts as measured by the Behavioral Risk Factor Surveillance System (BRFSS) during a period of increasing varicella vaccine coverage, 19982003. BMC Public Health 5:68.

Zhou, F. 2005. Economic Evaluation of the Universal Varicella Vaccination Program in the U.S. In Centers for Disease Control and Prevention, National Immunization Program, Record of the Meeting of the Advisory Committee on Immunization Practices, June 29-30, 2005, page 31. http://www.cdc.gov/NIP/ ACIP/minutes/acip-min-jun05.pdf.

Zhou, W., V. Pool, J. K. Iskander, R. English-Bullard, R. Ball, R. P. Wise, et al. 2003. Surveillance for safety after immunization: Vaccine Adverse Event Reporting System (VAERS)-United States, 1991-2001. MMWR Surveill. Summ. 52:1-24. 